\title{
Tofacitinib protects intestinal epithelial cells against oxygen-glucose deprivation/reoxygenation injury by inhibiting the JAK/STAT3 signaling pathway
}

\author{
JING YANG and XIAOLI XIE \\ Department of Pediatric Gastroenterology, Chengdu Women's and Children's Central Hospital, \\ School of Medicine, University of Electronic Science and Technology of China, Chengdu, Sichuan 610091, P.R. China
}

Received March 3, 2021; Accepted June 30, 2021

DOI: $10.3892 /$ etm.2021.10542

\begin{abstract}
The present study aimed to investigate the role and potential mechanism of action of tofacitinib (Tofa) in intestinal ischemia/reperfusion (I/R) injury. The normal rat small intestine epithelial cell line, IEC-6, was used to establish an I/R injury model by inducing oxygen-glucose deprivation/reoxygenation (OGD/R). Cells were divided into the following five groups: Control, OGD/R, OGD/R with 50, 100 and $200 \mathrm{nM}$ Tofa. Following Tofa administration, cell viability was measured using Cell Counting Kit- 8 assay and a lactate dehydrogenase detection kit. The expression levels of cell apoptosis-related proteins, Bcl-2, cleaved-caspase- 3 and cleaved-caspase- 9 were detected using western blot analysis. Additionally, the levels of oxidative stress-related markers, such as reactive oxygen species (ROS), malondialdehyde (MDA) and superoxide dismutase (SOD), and inflammatory cytokines, TNF- $\alpha$, IL- 6 and IL- $1 \beta$ were assessed using the colorimetric method. Western blot analysis was also used to measure the expression levels of the Janus kinase (JAK)/STAT3 signaling pathway-related proteins, including phosphorylated (p)-JAK1, p-JAK3 and p-STAT3. Subsequently, colivelin, an agonist of the JAK/STAT3 pathway, was used to investigate whether the effects of Tofa on intestinal I/R injury were mediated by this signaling pathway. The results showed that Tofa dose-dependently elevated cell viability compared with that in the OGD/R group. By contrast, Tofa attenuated cell apoptosis, which was coupled with upregulated Bcl-2 expression, downregulated cleaved-caspase-3 and downregulated cleaved-caspase-9 levels, in OGD/R-induced IEC- 6 cells . Furthermore, the contents of ROS and MDA were significantly
\end{abstract}

Correspondence to: Dr Xiaoli Xie, Department of Pediatric Gastroenterology, Chengdu Women's and Children's Central Hospital, School of Medicine, University of Electronic Science and Technology of China, 1617 Riyue Road, Chengdu, Sichuan 610091, P.R. China

E-mail: xiexiaolxl@163.com

Key words: intestinal ischemia/reperfusion, apoptosis, oxidative stress, inflammation, Janus kinase increased following exposure to OGD/R, which were accompanied by the decreased activity of SOD. These effects were reversed following cell treatment with Tofa. Consistently, Tofa intervention reduced the secretion levels of TNF- $\alpha$, IL-6 and IL-1 $\beta$ in a dose-dependent manner. Additionally, Tofa markedly downregulated the phosphorylation levels of JAK1, JAK3 and STAT3 in OGD/R-induced IEC-6 cells. However, treatment with colivelin markedly reversed the inhibitory effects of Tofa on cell viability, cell apoptosis, oxidative stress and inflammation. Overall, the findings of the present study suggested that Tofa could protect against intestinal I/R injury by inhibiting the JAK/STAT3 signaling pathway, which may hold promise as a therapeutic agent for intestinal I/R injury.

\section{Introduction}

The small intestinal mucosa functions as a protective barrier against various environmental stimuli and is considered to be the most sensitive and vulnerable site of ischemia/reperfusion (I/R) injury (1). Intestinal I/R injury typically occurs after acute mesenteric ischemia, small bowel volvulus, major surgical procedures, hemorrhagic shock and sepsis $(2,3)$. The development of intestinal $\mathrm{I} / \mathrm{R}$ is associated with intestinal mucosa injury, serious impairment of the local microvasculature, increased vascular and mucosal permeability and multiple organ failure, all of which contribute to increasing the risk of mortality $(4,5)$. To date, although significant improvements have been achieved in medical and surgical techniques, including hyperbaric oxygen treatment, ulinastatin and sodium pyruvate intravenous resuscitation combined with abdominal resuscitation, intestinal $\mathrm{I} / \mathrm{R}$ injury remains to be a serious clinical challenge (6-8).

A growing body of evidence has shown that the oxidative stress response, inflammation and cell apoptosis are involved in the progression of intestinal I/R, which can lead to the disruption of the intestinal barrier and distant organ injury $(9,10)$. Previous studies have proposed that I/R-induced production of reactive oxygen species (ROS) and apoptosis can cause damage to DNA and other cellular biomolecules, such as proteins and saccharides (11-13). In addition, activation of inflammation induced by ROS, which releases inflammatory cytokines and oxygen-derived free radicals, can aggravate intestinal injury 
further $(14,15)$. It has been reported that tofacitinib (Tofa), an oral small-molecule Janus kinase (JAK) inhibitor, is a potentially effective inductive therapeutic option for patients with moderate-to-severe ulcerative colitis (16). Additionally, Tofa was found to inhibit T-cell homing and activation during chronic intestinal inflammation in an experimental mouse colitis model (17), whereas another study revealed that Tofa could rescue human intestinal epithelial cells and colonoids from interferon- $\gamma$-induced barrier dysfunction (18). Tofa has also been shown to suppress the activities of all JAKs, particularly JAK1 and JAK3 in mammalian inflammatory bowel disease $(19,20)$. JAK1 and JAK3 can activate STATs by phosphorylation (21). An increasing number of studies have suggested that the JAK/STAT signaling is involved in the pathogenesis of tissue and organ I/R injury, including intestinal I/R injury (22-24). Therefore, the present study aimed to investigate the role of Tofa and its possible regulatory effects on the JAK/STAT signaling pathway in intestinal I/R injury.

In the present study, an oxygen-glucose deprivation/reoxygenation (OGD/R)-induced normal rat small intestinal epithelial cell model was established to simulate the physiological environment of intestinal I/R injury. The effects of Tofa on oxidative stress, inflammation and apoptosis caused by $\mathrm{OGD} / \mathrm{R}$-induced intestinal I/R injury were investigated and the regulatory mechanisms associated with JAK/STAT3 signaling pathway were explored.

\section{Materials and methods}

Cell culture and treatment. The normal rat small intestinal epithelial cell line, IEC-6, was obtained from the American Type Culture Collection. Cells were routinely cultured in DMEM (Gibco; Thermo Fisher Scientific, Inc.) supplemented with $10 \%$ FBS (Gibco; Thermo Fisher Scientific, Inc.) in a $5 \% \mathrm{CO}_{2}$ incubator at $37^{\circ} \mathrm{C}$. Tofa was purchased from Selleckchem. Tofa was dissolved in $100 \%$ dimethyl sulfoxide (DMSO; Sigma-Aldrich, Merck $\mathrm{KGaA}$ ) to a stock concentration of $10 \mathrm{mM}$ and stored at $-20^{\circ} \mathrm{C}$ until use as the vehicle. For 50, 100 and $200 \mathrm{nM}$ Tofa concentrations, the stock was diluted using DMEM. After reaching $80 \%$ confluence, cells were pretreated with $0.5 \mu \mathrm{M}$ colivelin (Shanghai Aladdin Biochemical Technology Co., Ltd.) for $6 \mathrm{~h}$ at $37^{\circ} \mathrm{C}$ and were then incubated in the presence of different doses of Tofa (50, 100 or $200 \mathrm{nM}$ ) for an additional $24 \mathrm{~h} 37^{\circ} \mathrm{C}$. Colivelin, an agonist of the JAK/STAT3 pathway, was used to investigate whether the effects of Tofa on intestinal I/R injury were mediated by this signaling pathway (25).

Construction of the $O G D / R$ model. Briefly, IEC-6 cells were first grown under normal conditions until they reached $80 \%$ confluence. Subsequently, the DMEM was replaced with D-Hanks buffer (Sigma-Aldrich; Merck KGaA) and cells were incubated in a modular incubator chamber filled with a $95 \% \mathrm{~N}_{2}$ and $5 \% \mathrm{CO}_{2}$ gas mixture for $4 \mathrm{~h}$ at $37^{\circ} \mathrm{C}$. The medium was then changed back to DMEM supplemented with $10 \%$ FBS and cells were reoxygenated for $4 \mathrm{~h}$. Control cells were not deprived of oxygen and glucose and were maintained in complete DMEM in a fully oxygenated environment.

Cell viability assay and lactate dehydrogenase ( $L D H)$ detection. Cell viability was assessed using a Cell Counting
Kit-8 (CCK-8) reagent (Shanghai YiSheng Biotechnology Co., Ltd.) according to manufacturer's protocol. Briefly, $3 \times 10^{3}$ IEC- 6 cells were plated into 96-well culture plates. At the indicated time, $10 \mu \mathrm{l} \mathrm{CCK}-8$ solution was added into each well and cells were incubated at $37^{\circ} \mathrm{C}$ for $2 \mathrm{~h}$. The absorbance values in each well were recorded at a wavelength of $450 \mathrm{~nm}$ using a microplate reader (Bio-Rad Laboratories, Inc.).

Furthermore, cell death was evaluated by measuring the activity of LDH in the supernatant using a LDH assay kit (cat. no. A020-2-2; Nanjing Jiancheng Bioengineering Institute) according to the manufacturer's instructions.

Detection of intracellular ROS. The production of intracellular ROS in IEC-6 cells was detected utilizing a ROS assay kit (cat. no. S0033; Beyotime Institute of Biotechnology) with cell-permeable 2',7'-dichlorodihydrofluorescein diacetate (DCFH-DA), according to the manufacturer's protocol. Briefly, following OGD/R induction, cells were stained with DCFH-DA $(10 \mu \mathrm{mol} / \mathrm{l})$ and DAPI $(1 \mu \mathrm{g} / \mathrm{l})$ for $15 \mathrm{~min}$ in the dark at $37^{\circ} \mathrm{C}$. The fluorescence intensity of ROS was measured under a confocal microscope (magnification, x200; Olympus Corporation) using the excitation and emission wavelengths of 488 and $530 \mathrm{~nm}$, respectively. ROS activity was quantified using ImageJ software (version 1.45; National Institutes of Health).

Measurement of oxidative stress-related factors. Following cell treatment, the content of malondialdehyde (MDA; cat. no. A003-4-1) and the activity of superoxide dismutase (SOD; cat. no. A001-3-2) in the culture media were detected using commercially available kits according to the protocols provided by the supplier (Nanjing Jiancheng Bioengineering Institute).

Determination of the secretion levels of inflammatory factors. After the end of the reoxygenation stage, the culture supernatants were collected. The concentration of inflammatory cytokines TNF- $\alpha$ (cat. no. F16960), IL-6 (cat. no. F15870) and IL-1 $\beta$ (cat. no. F15810), was determined using ELISA according to the manufacturer's protocol (Shanghai Xitang Biotechnology Co., Ltd.). Briefly, the treated cells were harvested by centrifugation at $4^{\circ} \mathrm{C}, 12,000 \mathrm{x} \mathrm{g}$ for $10 \mathrm{~min}$. The supernatant was then collected and plated into ELISA microplates to measure the absorbance of each well at a wavelength of $450 \mathrm{~nm}$ using an automatic microplate reader (Syngene).

Western blot analysis. Following treatment, IEC-6 cells were harvested and the cellular lysates were extracted with RIPA lysis buffer (Beyotime Institute of Biotechnology). The protein concentration in each group was measured using a BCA Protein Assay Kit (Beyotime Institute of Biotechnology). The proteins (40 $\mu \mathrm{g} /$ lane) were separated by $10 \%$ SDS-PAGE and were then transferred onto nitrocellulose membranes (Merck KGaA). Following incubation with 5\% non-fat milk for $1 \mathrm{~h}$ at room temperature, the membranes were probed with the following primary antibodies overnight at $4^{\circ} \mathrm{C}$ : Anti-Bcl-2 (1:1,000; cat. no. sc-7382; Santa Cruz Biotechnology, Inc.), anti-cleaved caspase-3 (1:1,000; cat. no. 9664T; Cell Signaling Technology, Inc.), anti-caspase-3 (1:1,000; cat. no. 9662S; Cell Signaling Technology, Inc.), anti-cleaved caspase-9 (1:1,000; cat. no. 20750S; Cell Signaling Technology, Inc.), anti-caspase-9 

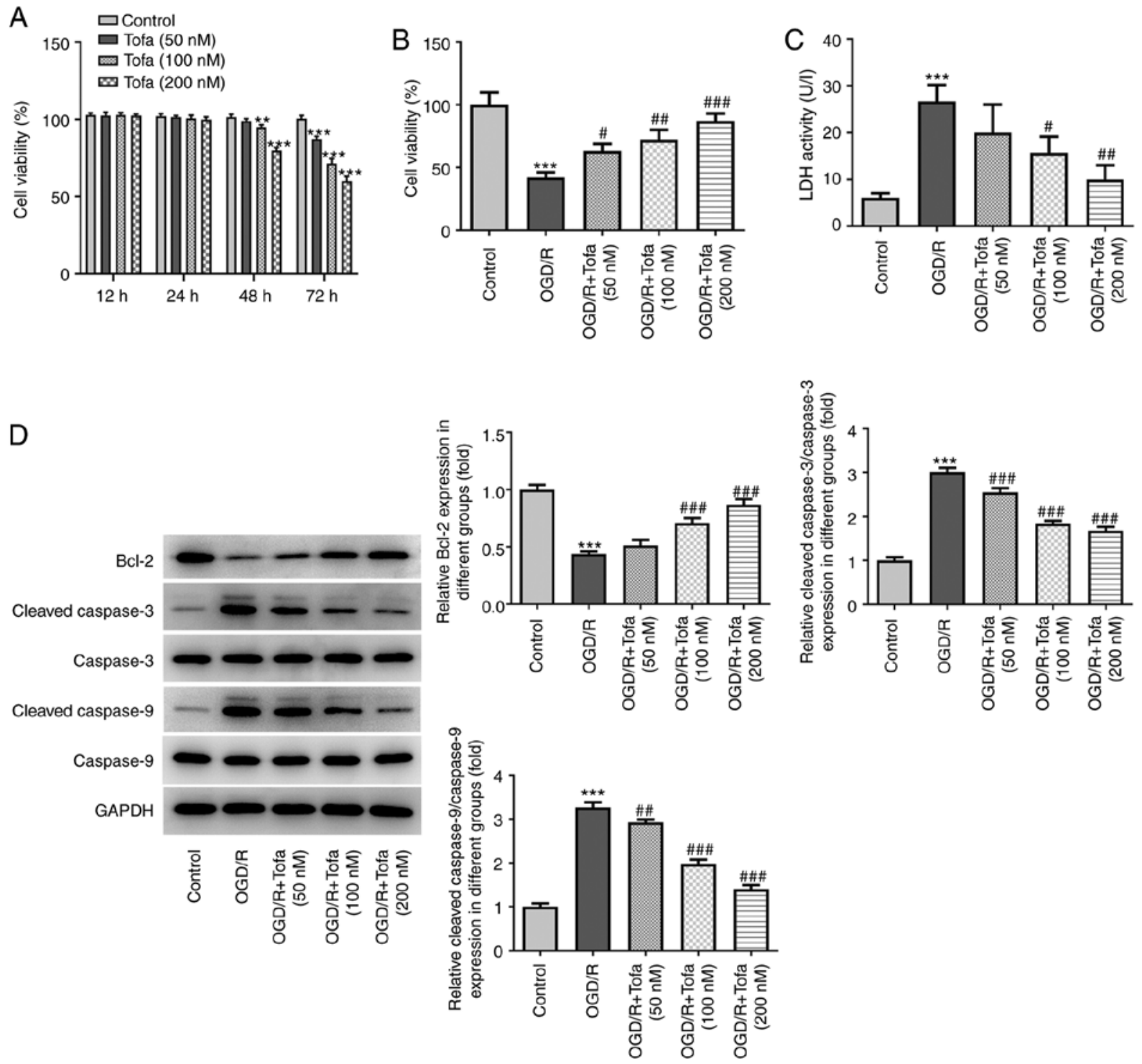

Figure 1. Tofa enhances viability and attenuates apoptosis in OGD/R-induced IEC-6 cells. (A) CCK-8 assay was used to detect IEC-6 cell viability after treatment with different doses of Tofa for $12,24,48$ and $72 \mathrm{~h}$. ${ }^{* *} \mathrm{P}<0.01$ and ${ }^{* * * *} \mathrm{P}<0.001$ vs. Control. (B) Cell viability was assessed by CCK-8 assay after treatment with different doses of Tofa for $24 \mathrm{~h}$ in OGD/R-induced IEC-6 cells. (C) LDH activity in the culture supernatant was measured using an LDH assay kit. (D) Western blot analysis was applied to detect the expression levels of apoptosis-related proteins. ${ }^{* * *} \mathrm{P}<0.001$ vs. Control. ${ }^{~} \mathrm{P}<0.05$; ${ }^{\# \#} \mathrm{P}<0.01$ and

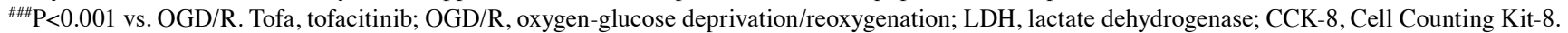

(1:1,000; cat. no. 9508T; Cell Signaling Technology, Inc.), anti-phosphorylated (p)-STAT3 (1:1,000; cat. no. 9145S; Cell Signaling Technology, Inc.), anti-STAT3 (1:1,000; cat. no. 4904T; Cell Signaling Technology, Inc.), anti-GAPDH (1:1,000; cat. no. 5174T; Cell Signaling Technology, Inc.), anti-p-JAK1 (1:1,000; cat. no. ab138005; Abcam), anti-JAK1 (1:1,000; cat. no. ab47435; Abcam), anti-p-JAK3 (1:1,000; cat. no. ab278789; Abcam) and anti-JAK3 (1:1,000; cat. no. ab45141; Abcam). Following primary incubation, the membranes were incubated with the corresponding secondary antibody conjugated with horseradish peroxidase (1:3,000; cat. no. 7074S; Cell Signaling Technology, Inc.) for $1 \mathrm{~h}$ at room temperature. The protein bands were visualized with using enhanced chemiluminescence substrate (Pierce; Thermo Fisher. Scientific, Inc.) in a chemiluminescence imaging equipment (Ultra-Lum, Inc.). Band intensity was semi-quantified via the ImageJ software (version 1.45; National Institutes of Health). GAPDH served as an internal control for normalization.
Statistical analysis. All experiments were repeated three times and the measurement data are expressed as the mean \pm SD. Data management and analysis were performed using the GraphPad Prism software (version 6.0; GraphPad Software, Inc.). Comparisons between two groups were conducted by an unpaired Student's t-test. One-way analysis of variance followed by Tukey's post hoc test was applied to compare differences among multiple groups. $\mathrm{P}<0.05$ was considered to indicate a statistically significant difference.

\section{Results}

Tofa treatment enhances the viability and attenuates apoptosis of OGD/R-induced IEC-6 cells. Firstly, IEC-6 cell viability was evaluated using a CCK-8 kit after treatment with Tofa $(50,100$ and $200 \mathrm{nM})$ for 24,48 and $72 \mathrm{~h}$. As shown in Fig. 1A, none of the doses of Tofa exerted significant effects 
A

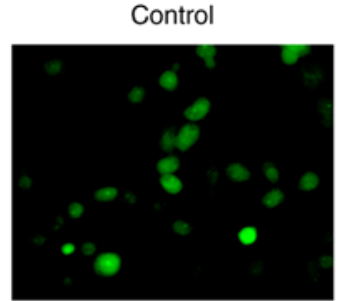

B

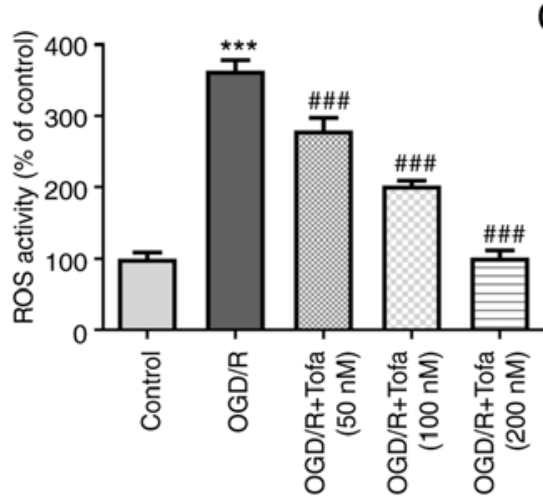

$\mathrm{OGD} / \mathrm{R}$

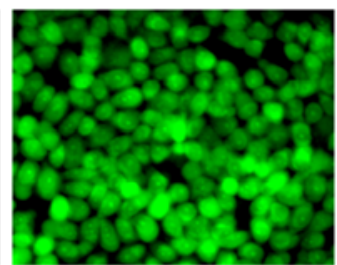

C

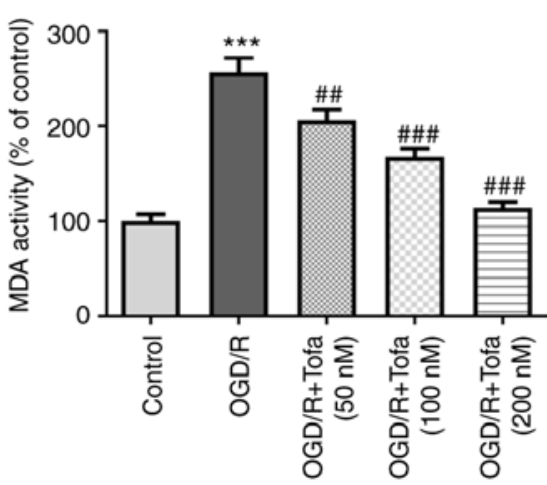

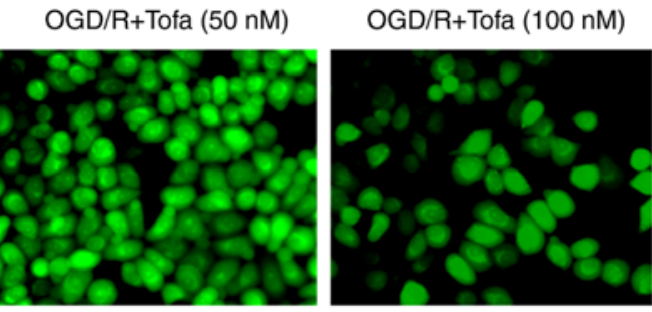

D
E

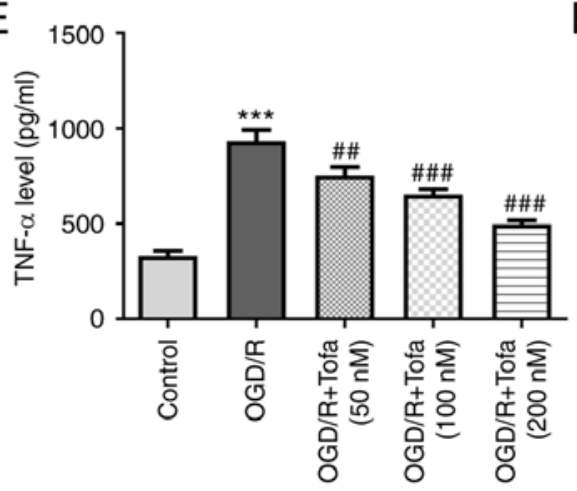

F

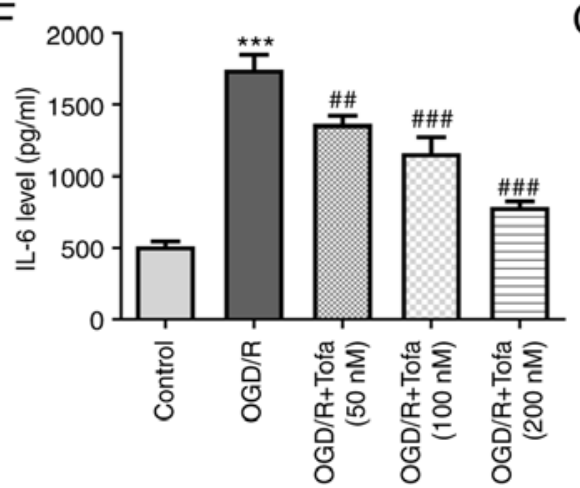

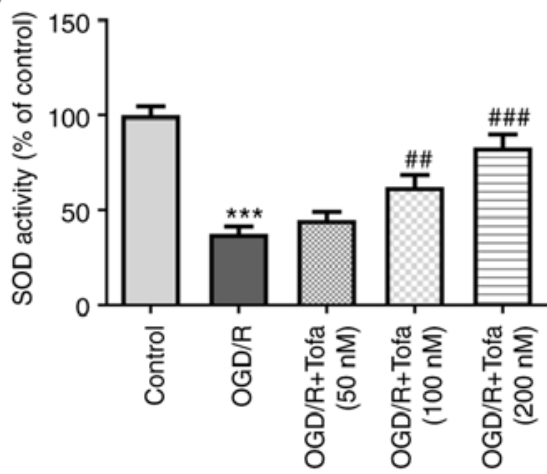

OGD/R+Tofa $(200 \mathrm{nM})$

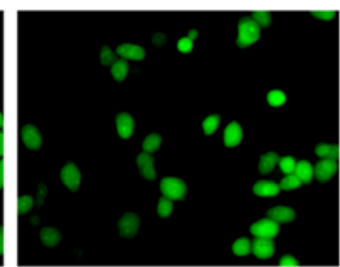

G

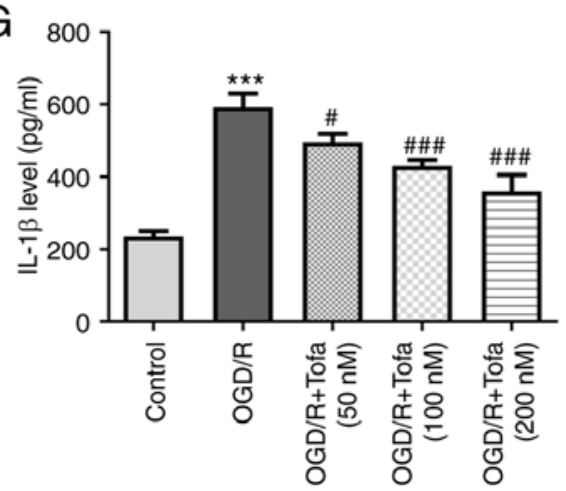

Figure 2. Tofa treatment attenuates oxidative stress and inflammation in IEC-6 cells that were exposed to OGD/R. (A) Intracellular ROS production was measured using 2',7'-dichlorodihydrofluorescein diacetate as a fluorescence probe, (B) which was quantified. Magnification, x200. (C) MDA content and (D) SOD activity were assessed in the cell culture supernatant by corresponding MDA and SOD assay kits. Secretion levels of inflammatory factors, namely (E) TNF- $\alpha$, (F) IL-6 and (G) IL-1 $\beta$, were measured using ELISA. ${ }^{* * *} \mathrm{P}<0.001$ vs. Control; ${ }^{*} \mathrm{P}<0.05$, ${ }^{\# \#} \mathrm{P}<0.01$ and ${ }^{\# \# \#} \mathrm{P}<0.001$ vs. OGD/R. Tofa, tofacitinib; $\mathrm{OGD} / \mathrm{R}$, oxygen-glucose deprivation/reoxygenation; ROS, reactive oxygen species; MDA, malondialdehyde; SOD, superoxide dismutase.

on cell viability after Tofa treatment for 12 and $24 \mathrm{~h}$. However, 100 and $200 \mathrm{nM}$ Tofa intervention for $48 \mathrm{~h}$, in addition to 50,100 and $200 \mathrm{nM}$ Tofa treatment for $72 \mathrm{~h}$, significantly decreased cell viability compared with that in the Control group. Therefore, treatment of IEC-6 cells with 50, 100 and $200 \mathrm{nM}$ for $24 \mathrm{~h}$ was chosen to be the regimen used for subsequent analyzes. Following Tofa preconditioning in IEC-6 cells exposed to OGD/R, cell viability was assessed using CCK-8 assay. As shown in Fig. 1B, OGD/R challenge significantly attenuated cell viability compared with that in the untreated control group. By contrast, Tofa treatment dose-dependently elevated IEC-6 cell viability compared with that in the OGD/R group. Additionally, compared with that in the OGD/R-induced group, cell treatment with 100 and $200 \mathrm{nM}$ Tofa markedly reduced the activity of LDH in the culture supernatant (Fig. 1C). According to Fig. 1D, the expression levels of Bcl-2 were significantly downregulated, whilst those of cleaved-caspase-3/9 were significantly upregulated, in the OGD/R group compared with those in the control group. However, these effects aforementioned were markedly reversed following Tofa pretreatment in a dose-dependent manner (Fig. 1D). These findings suggest that Tofa treatment can enhance cell viability whilst inhibiting apoptosis in OGD/R-induced IEC-6 cells.

Tofa treatment alleviates oxidative stress and inflammation in OGD/R-treated IEC-6 cells. To investigate the effects of Tofa on oxidative stress in OGD/R-treated IEC-6 cells, the production of intracellular ROS was measured. The results demonstrated that exposure to OGD/R significantly increased the intracellular production of ROS compared with that in the control group, whereas Tofa intervention dose-dependently and significantly decreased ROS levels in OGD/R-treated IEC-6 cells (Fig. 2A and B). Consistently, the content of MDA was also significantly enhanced after OGD/R challenge, accompanied by significantly reduced SOD activity, compared with those in the control group (Fig. 2C and D). However, these effects were dose-dependently reversed following treatment with Tofa. Furthermore, Tofa dose-dependently reversed the significant inflammatory responses in IEC-6 cells induced by OGD/R, as indicated by the significantly decreased concentrations of 

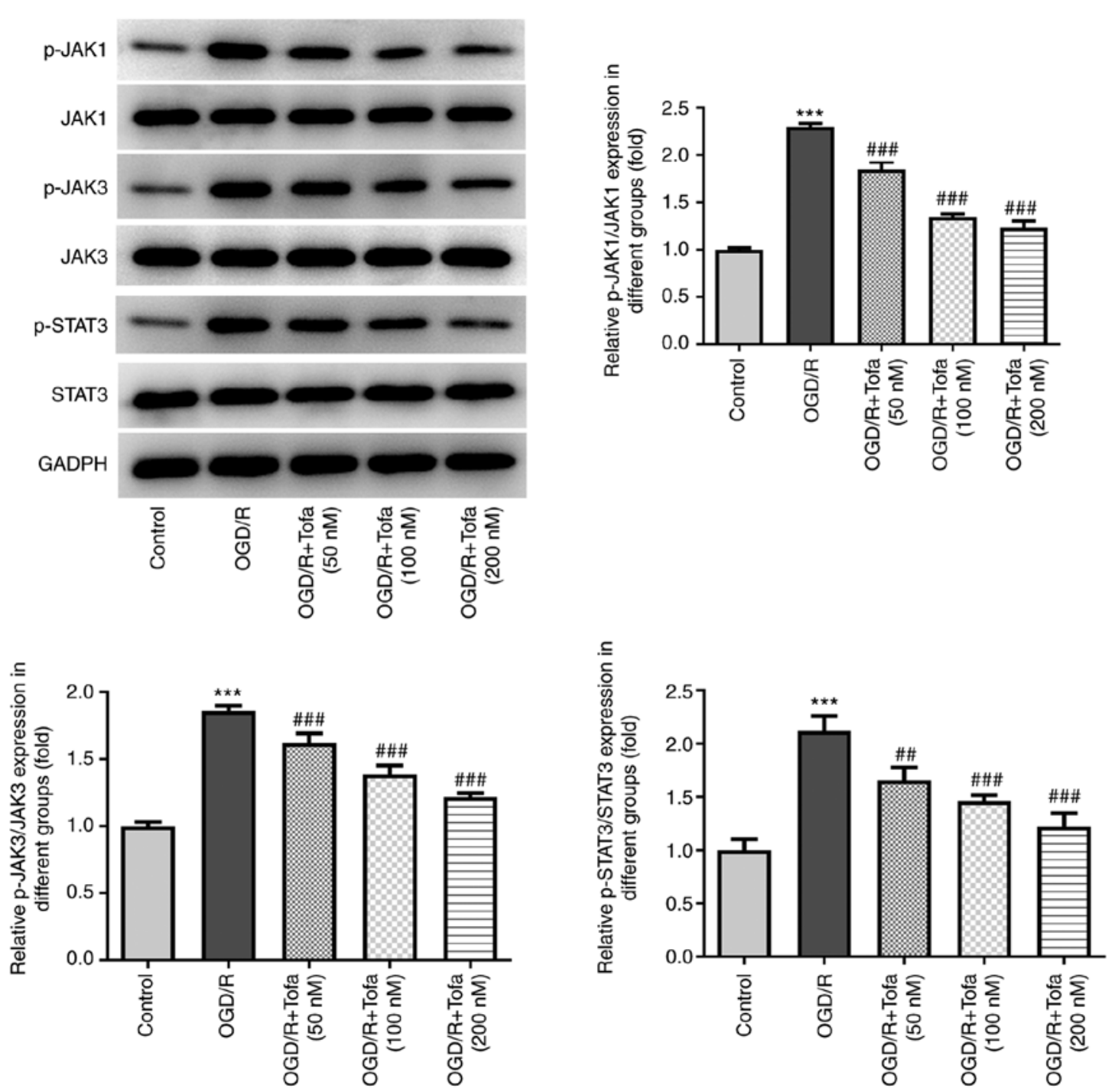

Figure 3. Tofa preconditioning inhibits JAK/STAT3 signaling in OGD/R-induced IEC-6 cells. The expression levels of JAK/STAT3 signaling pathway-related proteins were determined by western blot analysis. ${ }^{* * *} \mathrm{P}<0.001$ vs. Control; ${ }^{\# \#} \mathrm{P}<0.01$ and ${ }^{\# \# \#} \mathrm{P}<0.001$ vs. OGD/R. Tofa, tofacitinib; JAK, Janus kinase; OGD/R, oxygen-glucose deprivation/reoxygenation; p-, phosphorylated.

TNF- $\alpha$, IL-6 and IL-1 $\beta$ (Fig. 2E-G). These findings suggest that Tofa exert inhibitory effects on OGD/R-induced oxidative stress and inflammation in IEC-6 cells.

Tofa preconditioning inhibits JAK/STAT3 signaling in $O G D / R$-stimulated IEC- 6 cells. To investigate the potential mechanism of Tofa in OGD/R-induced IEC- 6 cells, the expression levels of the JAK/STAT3 signaling pathway-related proteins were detected by the means of western blot analysis. $\mathrm{OGD} / \mathrm{R}$ challenge significantly upregulated the levels of phosphorylated (p)-JAK1, p-JAK3 and p-STAT3 compared with those in the control group (Fig. 3). By contrast, Tofa treatment dose-dependently and significantly downregulated the phosphorylation of JAK1, JAK3 and STAT3 compared with those in the OGD/R group (Fig. 3). These findings suggest that Tofa can inhibit the JAK/STAT3 signaling pathway in OGD/R-induced IEC- 6 cells. effects of Tofa on OGD/R-induced IEC-6 cell injury. To verify the importance of the JAK/STAT3 pathway for the protective effects of Tofa on OGD/R-induced IEC- 6 cell injury, cells were treated with colivelin, an agonist of the JAK/STAT3 pathway (25). As shown in Fig. 4A, colivelin significantly abrogated the therapeutic effects of Tofa on cell viability after exposure to OGD/R. Additionally, significantly enhanced LDH activity was observed in the OGD/R + Tofa + colivelin group compared with that in the OGD/R + Tofa + vehicle group (Fig. 4B). Colivelin also significantly reversed the effects of Tofa on the expression of Bcl-2, cleaved caspase- 3 and cleaved caspase-9 in OGD/R-induced IEC- 6 cells (Fig. 4C).

Subsequently, the contents of ROS and MDA were significantly enhanced after colivelin treatment, which was also accompanied by the significantly decreased activity of the antioxidant enzyme SOD, compared with those in the OGD/R + Tofa + vehicle group (Fig. 5A-D). In addition, colivelin partially but significantly counteracted the inhibitory effects of Tofa on the secretion levels of TNF- $\alpha$, IL- 6 and IL-1 $\beta$ (Fig. 5E-G). 

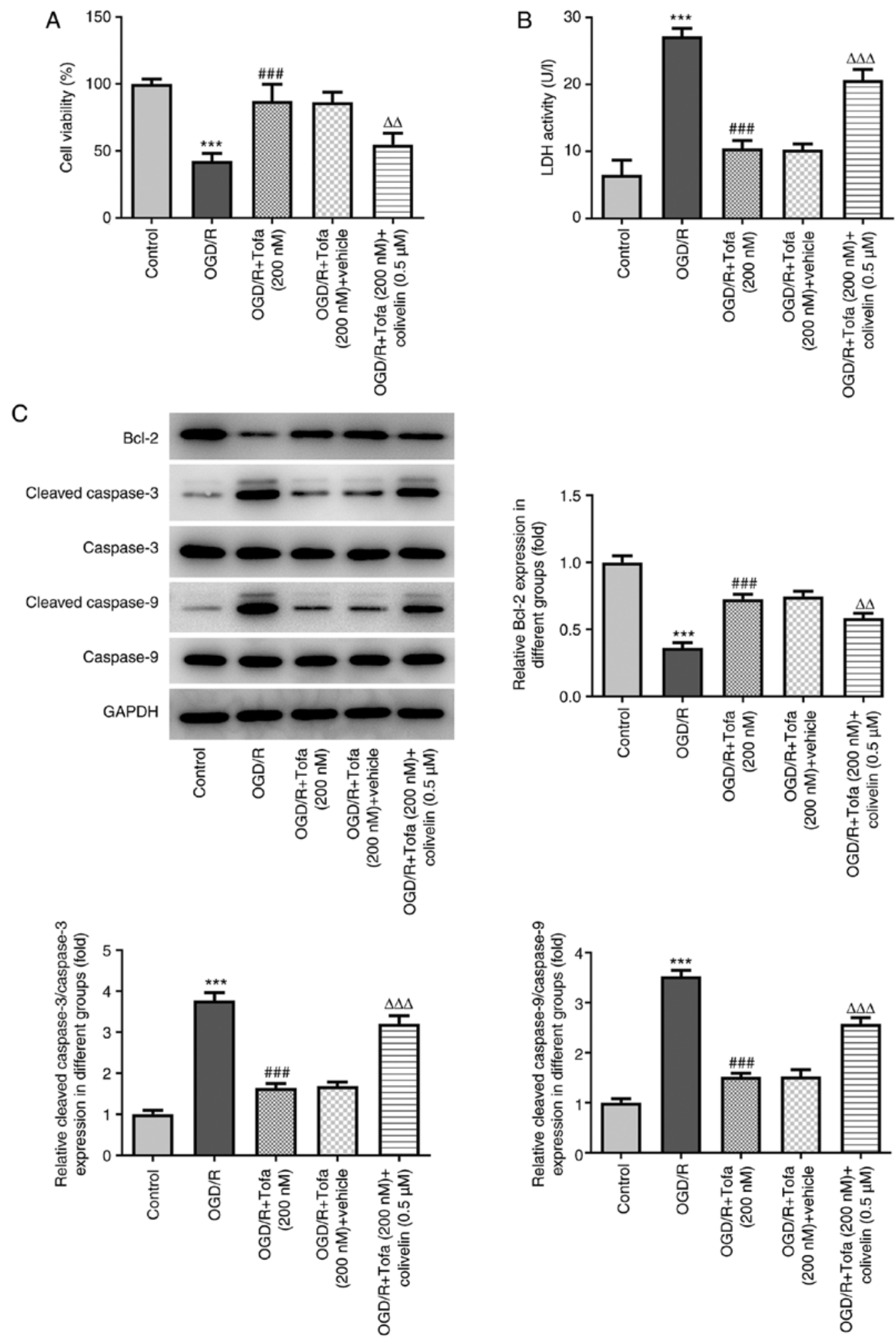

Figure 4. Activation of JAK/STAT3 signaling reverses the inhibitory effects of Tofa on OGD/R-induced IEC-6 cell apoptosis. (A) Cell viability was assessed using Cell Counting Kit- 8 assay in OGD/R-induced IEC- 6 cell apoptosis in the presence or absence of Tofa (200 nM) and colivelin (0.5 $\mu \mathrm{M})$. (B) LDH activity in the culture supernatant was determined using an LDH assay kit. (C) Western blot analysis was performed to determine the expression levels of apoptosis-related proteins. ${ }^{* * * *} \mathrm{P}<0.001$ vs. Control; ${ }^{\# \#} \mathrm{P}<0.001$ vs. OGD/R; ${ }^{\Delta \Delta} \mathrm{P}<0.01$ and ${ }^{\Delta \Delta \Delta} \mathrm{P}<0.001$ vs. OGD/R + Tofa + vehicle. JAK, Janus kinase; Tofa, tofacitinib; OGD/R, oxygen-glucose deprivation/reoxygenation; LDH, lactate dehydrogenase.

Consistent with the aforementioned observations, colivelin significantly upregulated the protein phosphorylation of JAK1, JAK3 and STAT3 (Fig. 6). Overall, these data suggest that the activation of JAK/STAT3 signaling could negate the effects of Tofa on OGD/R-induced IEC-6 cell injury.

\section{Discussion}

Intestinal I/R injury caused by the clamping of the superior mesenteric artery is a common life-threatening complication that can be observed in multiple clinical conditions, such as 
A

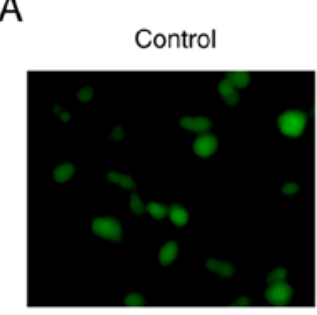

B
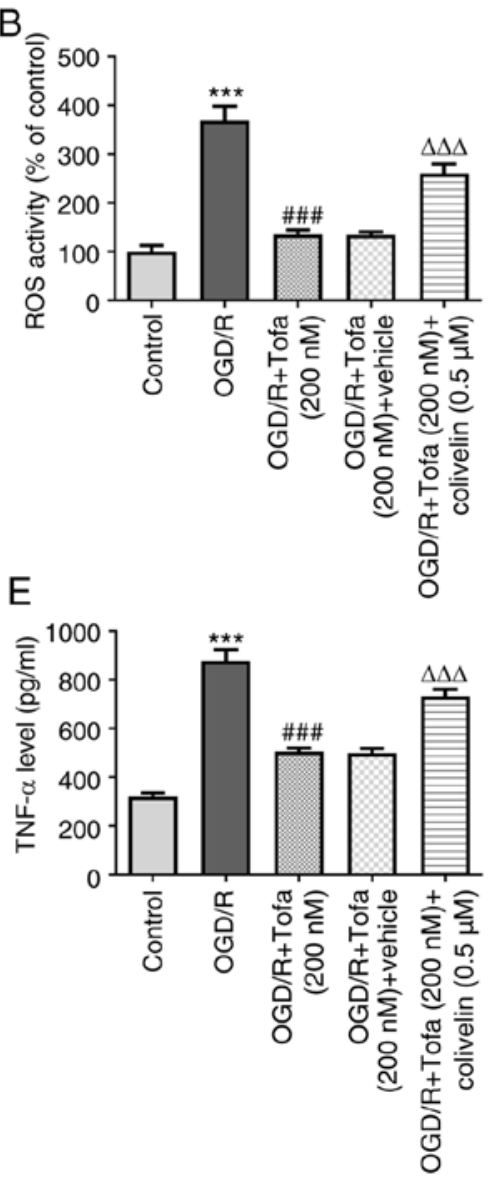

OGD/R

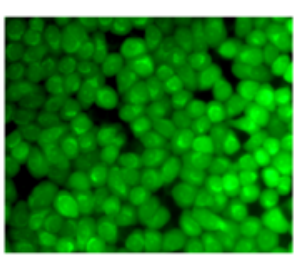

OGD/R+Tofa $(200 \mathrm{nM})$

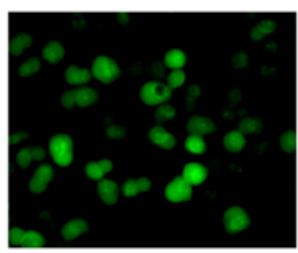

C

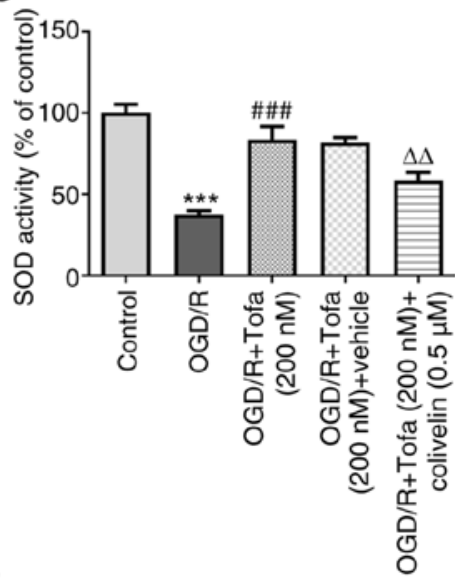

$\mathrm{F}$

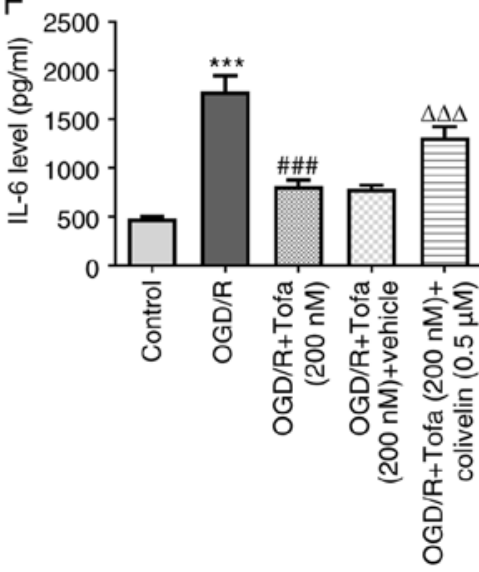

OGD/R+Tofa (200 nM)+vehicle

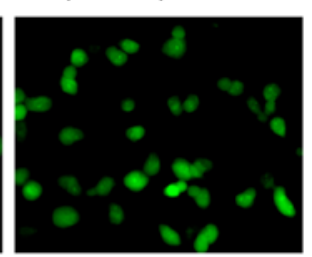

OGD/R+Tofa (200 nM)+ colivelin $(0.5 \mu \mathrm{M})$

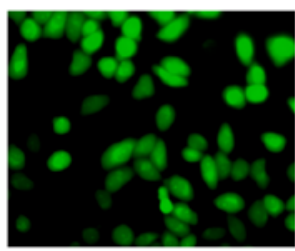

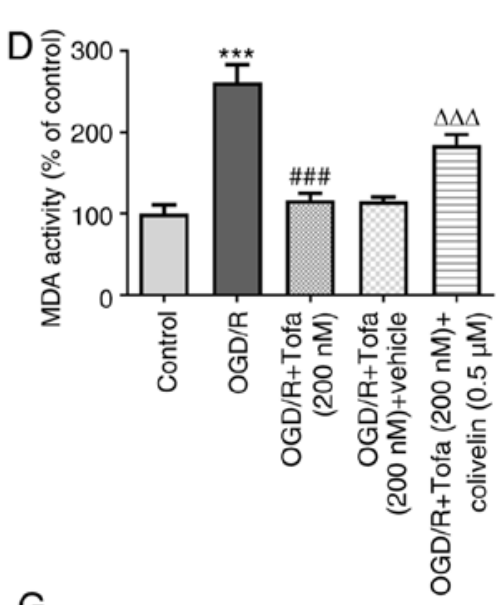

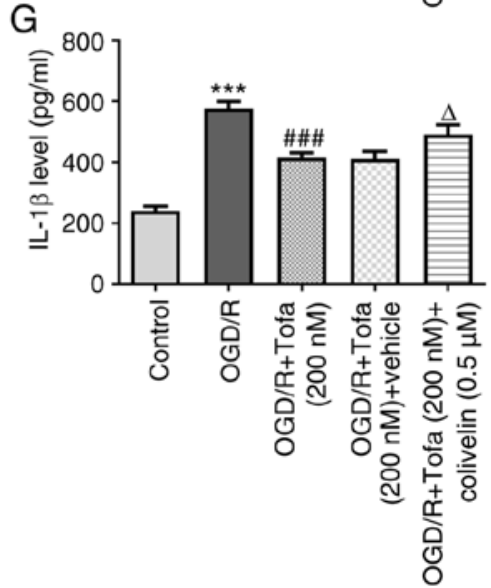

Figure 5. Activation of the JAK/STAT3 signaling reverses the inhibitory effects of Tofa on oxidative stress and inflammation in OGD/R-induced IEC-6 cells. (A) The levels of ROS were measured using 2',7'-dichlorodihydrofluorescein diacetate as a fluorescence probe, (B) which were quantified in OGD/R-induced IEC-6 cell apoptosis in the presence or absence of Tofa $(200 \mathrm{nM})$ and colivelin $(0.5 \mu \mathrm{M})$. Magnification, $\mathrm{x} 200$. The activity of (C) SOD and (D) the content of MDA in the cell culture supernatant were measured using corresponding MDA and SOD assay kits. ELISA was used to measure the concentration of (E) TNF- $\alpha$, (F) IL-6 and (G) IL-1 $\beta$. ${ }^{* * *} \mathrm{P}<0.001$ vs. Control; ${ }^{\# \#} \mathrm{P}<0.001$ vs. OGD/R; ${ }^{\Delta} \mathrm{P}<0.05,{ }^{\Delta \Delta} \mathrm{P}<0.01$ and ${ }^{\Delta \Delta \Delta} \mathrm{P}<0.001$ vs. OGD/R + Tofa + vehicle. JAK, Janus kinase; Tofa, tofacitinib; OGD/R, oxygen-glucose deprivation/reoxygenation; ROS, reactive oxygen species; SOD, superoxide dismutase; MDA, malondialdehyde.

small intestinal volvulus, acute mesenteric ischemia, shock and trauma (26). The present study provided useful findings using an OGD/R IEC-6 cell model to simulate the physiological environment during intestinal I/R injury. The results demonstrated that Tofa preconditioning exerted protective effects on apoptosis, oxidative stress and inflammation in IEC- 6 cells in a dose-dependent manner during OGD/R. Mechanically, the aforementioned beneficial effects of Tofa were partially abrogated by the agonist of the JAK/STAT3 pathway.

A growing body of evidence suggests that intestinal I/R injury is characterized by disruption of the mucosal barrier, which may result in systemic inflammatory response syndrome and multiple organ failure (27-29). Apoptosis is a major mechanism of mucosal epithelial cell death during intestinal I/R-induced destruction of the intestinal epithelial barrier (30). Consistent with the results of the present study, a previous study demonstrated that OGD/R challenge markedly enhanced the apoptosis of IEC-6 cells (31). Additionally, intestinal I/R injury is caused by oxidative damage due to the imbalance in oxidation and antioxidation in ischemic tissues and cells, such that ROS cannot be removed efficiently after blood supply is restored (32). MDA represents one of the end products of lipid peroxidation and is an oxidative stress marker, whereas SOD is a crucial antioxidant enzyme that is part of the defense system against oxidative stress and can protect intestinal epithelial cells against ROS-induced cell death $(33,34)$. Tofa has been reported to inhibit the production of ROS triggered by oxidized low-density lipoprotein in cultured primary human aortic endothelial cells (35). In the present 

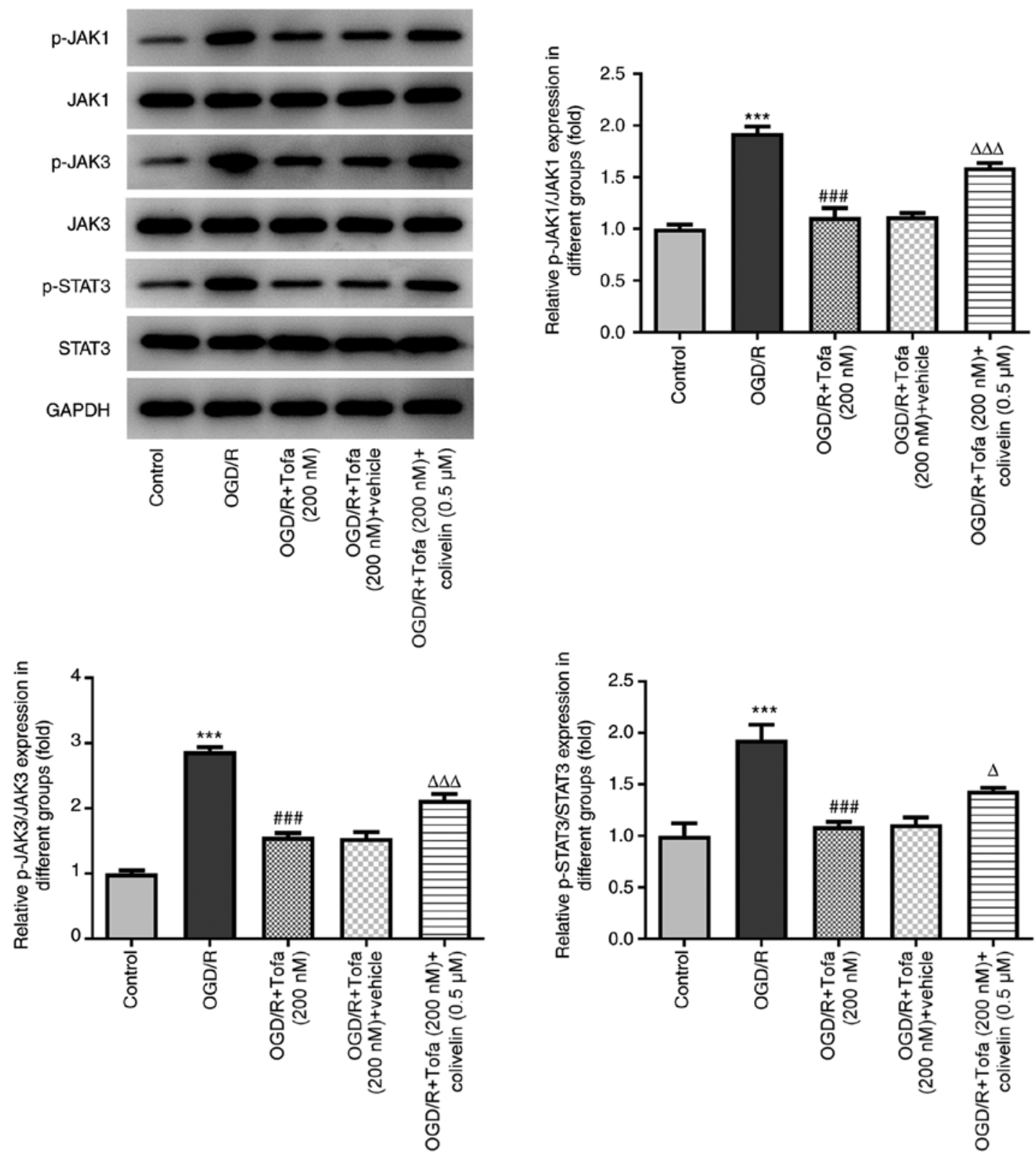

Figure 6. Colivelin treatment activates the JAK/STAT3 signaling pathway in OGD/R-induced IEC-6 cells. Western blotting was used to determine the expression levels of the JAK/STAT3 signaling pathway-related proteins in OGD/R-induced IEC- 6 cell apoptosis in the presence or absence of Tofa (200 nM) and colivelin $(0.5 \mu \mathrm{M}) .{ }^{* * * *} \mathrm{P}<0.001$ vs. Control; ${ }^{\# \# \#} \mathrm{P}<0.001$ vs. OGD/R; ${ }^{\Delta} \mathrm{P}<0.05$ and ${ }^{\Delta \Delta \Delta} \mathrm{P}<0.001$ vs. OGD/R + Tofa + vehicle. JAK, Janus kinase; OGD/R, oxygen-glucose deprivation/reoxygenation; Tofa, tofacitinib; p-, phosphorylated.

study, Tofa alleviated oxidative stress in OGD/R-induced intestinal epithelial cells, as indicated by the reduced levels of ROS and MDA and enhanced activity of SOD, which were in accordance with previous studies $(15,36)$. Emerging evidence has suggested that the predominant cause of intestinal $\mathrm{I} / \mathrm{R}$ damage is the excessive release of inflammatory factors $(14,37)$. In addition, it has been reported that during intestinal I/R, the levels of proinflammatory cytokines, including TNF- $\alpha$, IL- 6 and IL-1 $\beta$, are notably enhanced, which contribute to the induction of the systemic inflammatory response and even damage of distant organs (38). Tofa is a well-known small-molecule JAK inhibitor that has been approved for the treatment of rheumatoid arthritis (39). Furthermore, Tofa is recommended for the treatment of adult ulcerative colitis, which is a type of inflammatory bowel disease (40). Tofa can suppress T-cell homing and activation during chronic intestinal inflammation (17) and rescue human intestinal epithelial cells and colonoids from cytokine-induced barrier dysfunction (18). The present study revealed that Tofa could dose-dependently alleviate OGD/R-induced IEC- 6 cell damage, suggesting the potential use of Tofa for treating intestinal I/R injury.

The present study also investigated the role of the JAK/STAT3 signaling in the protective effects of Tofa on OGD/R-induced IEC- 6 cell injury. To the best of our knowledge, Tofa is a well-known small-molecule JAK inhibitor, which inhibits all JAKs, particularly JAK1 and JAK3 $(19,20)$. JAK1 and JAK3 activate STATs by phosphorylation (21). It has been previously reported that JAK/STAT signaling is involved in several important cellular processes, including cell 
proliferation, migration, apoptosis and inflammation (41-43). A number of studies have suggested that JAK/STAT signaling is involved in the pathogenesis of tissue and organ I/R injury, including intestinal I/R injury (22-24). By inactivating JAK/STAT3 signaling, dexmedetomidine was shown to inhibit the apoptosis of astrocytes induced by OGD/R (44). Fish oils have also been found to provide protection against cecal ligation and puncture-induced septic acute kidney injury by regulating inflammation, oxidative stress and apoptosis by suppressing JAK/STAT3 signaling (45). The present study suggests that Tofa can dose-dependently downregulate the phosphorylation and therefore activation of JAK1, JAK2 and STAT3 in IEC-6 cells under OGD/R conditions. Importantly, colivelin, which is a JAK activator, partially counteracted the beneficial effects of Tofa on OGD/R-induced IEC-6 cell injury.

To conclude, to the best of our knowledge, the present study was the first to demonstrate that Tofa exerted anti-apoptotic, antioxidant and anti-inflammatory effects during intestinal I/R injury in vitro by inactivating the JAK/STAT3 signaling pathway. This suggests that Tofa may be a promising candidate for the treatment and prevention of intestinal I/R injury in the clinic. However, the lack of in vivo experiments using an intestinal I/R injury animal model is a limitation of the present study. Therefore, further intestinal I/R animal experiments to explore the potential effects of Tofa on I/R-induced intestinal damage should be performed in future studies to support the present conclusions.

\section{Acknowledgements}

Not applicable.

\section{Funding}

Not funding was received.

\section{Availability of data and materials}

The datasets used and/or analyzed during the current study are available from the corresponding author on reasonable request.

\section{Authors' contributions}

JY and XX searched the literature, designed and conducted the experiments. JY analyzed and interpreted the data and wrote the manuscript. XX revised the manuscript. JY and XX confirm the authenticity of all the raw data. All the authors have read and approved the final version of the manuscript.

\section{Ethics approval and consent to participate}

Not applicable.

\section{Patient consent for publication}

Not applicable.

\section{Competing interests}

The authors declare that they have no competing interests.

\section{References}

1. Li Y, Feng D, Wang Z, Zhao Y, Sun R, Tian D, Liu D, Zhang F, Ning S, Yao J and Tian X: Ischemia-induced ACSL4 activation contributes to ferroptosis-mediated tissue injury in intestinal ischemia/reperfusion. Cell Death Differ 26: 2284-2299, 2019.

2. Sun Y, Lian M, Lin Y, Xu B, Li Y, Wen J, Chen D, Xu M, Almoiliqy $M$ and Wang L: Role of p-MKK7 in myricetin-induced protection against intestinal ischemia/reperfusion injury. Pharmacol Res 129: 432-442, 2018.

3. Karhausen J, Bernstock JD, Johnson KR, Sheng H, Ma Q, Shen Y, Yang W, Hallenbeck JM and Paschen W: Ubc9 overexpression and SUMO1 deficiency blunt inflammation after intestinal ischemia/reperfusion. Lab Invest 98: 799-813, 2018.

4. Leone M, Bechis C, Baumstarck K, Ouattara A, Collange O, Augustin P, Annane D, Arbelot C, Asehnoune K, Baldési O, et al: Outcome of acute mesenteric ischemia in the intensive care unit: A retrospective, multicenter study of 780 cases. Intensive Care Med 41: 667-676, 2015.

5. Liu ZM, Zhang XY, Chen J, Shen JT, Jiang ZY and Guan XD: Terlipressin protects intestinal epithelial cells against oxygen-glucose deprivation/re-oxygenation injury via the phosphatidylinositol 3-kinase pathway. Exp Ther Med 14: 260-266, 2017.

6. Yu JS, Yan S, Liu XS, Wu YJ, Fu PF, Wu LH and Zheng SS: Attenuation of graft ischemia-reperfusion injury by urinary trypsin inhibitor in mouse intestinal transplantation. World $\mathbf{J}$ Gastroenterol 11: 1605-1609, 2005.

7. Daniel RA, Cardoso VK, Góis E Jr, Parra RS, Garcia SB, Rocha JJ and Féres O: Effect of hyperbaric oxygen therapy on the intestinal ischemia reperfusion injury. Acta Cir Bras 26: 463-469, 2011.

8. Miyake H, Koike Y, Seo S, Lee C, Li B, Ganji N and Pierro A: The effect of pre- and post-remote ischemic conditioning reduces the injury associated with intestinal ischemia/reperfusion. Pediatr Surg Int 36: 1437-1442, 2020.

9. Feinman R, Deitch EA, Watkins AC, Abungu B, Colorado I, Kannan KB, Sheth SU, Caputo FJ, Lu Q, Ramanathan M, et al: HIF-1 mediates pathogenic inflammatory responses to intestinal ischemia-reperfusion injury. Am J Physiol Gastrointest Liver Physiol 299: G833-G843, 2010.

10. Wang G, Yao J, Li Z, Zu G, Feng D, Shan W, Li Y, Hu Y, Zhao Y and Tian X: miR-34a-5p inhibition alleviates intestinal ischemia/reperfusion-induced reactive oxygen species accumulation and apoptosis via activation of SIRT1 signaling. Antioxid Redox Signal 24: 961-973, 2016.

11. Rodriguez-Lara SQ, Cardona-Muñoz EG, Ramirez-Lizardo EJ, Totsuka-Sutto SE, Castillo-Romero A, García-Cobián TA and García-Benavides L: Alternative interventions to prevent oxidative damage following ischemia/reperfusion. Oxid Med Cell Longev 2016: 7190943, 2016.

12. Pérez S, Taléns-Visconti R, Rius-Pérez S, Finamor I and Sastre J: Redox signaling in the gastrointestinal tract. Free Radic Biol Med 104: 75-103, 2017.

13. Ameli M, Hashemi MS, Moghimian M and Shokoohi M: Protective effect of tadalafil and verapamil on testicular function and oxidative stress after torsion/detorsion in adult male rat. Andrologia 50: e13068, 2018.

14. de Groot H and Rauen U: Ischemia-reperfusion injury: Processes in pathogenetic networks: A review. Transplant Proc 39: 481-484, 2007.

15. Zu G, Guo J, Che N, Zhou T, Zhang X, Wang G, Ji A and Tian X: Protective effects of ginsenoside Rg1 on intestinal ischemia/reperfusion injury-induced oxidative stress and apoptosis via activation of the Wnt/ $\beta$-catenin pathway. Sci Rep 6: 38480, 2016.

16. Sandborn WJ, Ghosh S, Panes J, Vranic I, Su C, Rousell S and Niezychowski W; Study A3921063 Investigators: Tofacitinib, an oral janus kinase inhibitor, in active ulcerative colitis. N Engl J Med 367: 616-624, 2012.

17. Lechner K, Gerlach K, Popp V, Offensperger L, Zundler S, Wiendl M, Becker E, Atreya R, Rath T, Neurath MF and Weigmann B: The JAK1/3 inhibitor tofacitinib suppresses T cell homing and activation in chronic intestinal inflammation. J Crohns Colitis: Aug 18, 2020 (Epub ahead of print).

18. Sayoc-Becerra A, Krishnan M, Fan S, Jimenez J, Hernandez R, Gibson K, Preciado R, Butt G and McCole DF: The JAK-inhibitor tofacitinib rescues human intestinal epithelial cells and colonoids from cytokine-induced barrier dysfunction. Inflamm Bowel Dis 26: 407-422, 2020. 
19. Flanagan ME, Blumenkopf TA, Brissette WH, Brown MF, Casavant JM, Shang-Poa C, Doty JL, Elliott EA, Fisher MB, Hines M, et al: Discovery of CP-690,550: A potent and selective janus kinase (JAK) inhibitor for the treatment of autoimmune diseases and organ transplant rejection. J Med Chem 53: 8468-8484, 2010.

20. Danese S, Grisham M, Hodge J and Telliez JB: JAK inhibition using tofacitinib for inflammatory bowel disease treatment: A hub for multiple inflammatory cytokines. Am J Physiol Gastrointest Liver Physiol 310: G155-G162, 2016.

21. Clark JD, Flanagan ME and Telliez JB: Discovery and development of janus kinase (JAK) inhibitors for inflammatory diseases J Med Chem 57: 5023-5038, 2014.

22. Zhang H, Liu X, Yang F, Cheng D and Liu W: Overexpression of HIF-1 $\alpha$ protects PC12 cells against OGD/R-evoked injury by reducing miR-134 expression. Cell Cycle 19: 990-999, 2020.

23. Fang H, Zhang FX, Li HF, Yang M, Liao R, Wang RR, Wang QY, Zheng PC and Zhang JP: PRR34-AS1 overexpression promotes protection of propofol pretreatment against ischemia/reperfusion injury in a mouse model after total knee arthroplasty via blockade of the JAK1-dependent JAK-STAT signaling pathway. J Cell Physiol 235: 2545-2556, 2020.

24. Zhang XK, Zhou XP, Zhang Q and Zhu F: The preventive effects of dexmedetomidine against intestinal ischemia-reperfusion injury in wistar rats. Iran J Basic Med Sci 18: 604-609, 2015.

25. Zhao H, Feng Y, Wei C, Li Y, Ma H, Wang X, Cui Z, Jin WN and Shi FD: Colivelin rescues ischemic neuron and axons involving JAK/STAT3 signaling pathway. Neuroscience 416: 198-206, 2019.

26. Zhou J, Huang WQ, Li C, Wu GY, Li YS, Wen SH, Lei WL and Liu KX: Intestinal ischemia/reperfusion enhances microglial activation and induces cerebral injury and memory dysfunction in rats. Crit Care Med 40: 2438-2448, 2012.

27. Vollmar B and Menger MD: Intestinal ischemia/reperfusion: Microcirculatory pathology and functional consequences. Langenbecks Arch Surg 396: 13-29, 2011.

28. Dai H, Wang M, Patel PN, Kalogeris T, Liu Y, Durante W and Korthuis RJ: Preconditioning with the $\mathrm{BK}_{\mathrm{Ca}}$ channel activator NS-1619 prevents ischemia-reperfusion-induced inflammation and mucosal barrier dysfunction: Roles for ROS and heme oxygenase-1. Am J Physiol Heart Circ Physiol 313: H988-H999, 2017.

29. Lin ZL, Tan SJ, Cheng MH, Zhao CY, Yu WK, He YL, Li J and $\mathrm{Li}$ N: Lipid-rich enteral nutrition controls intestinal inflammation, improves intestinal motility and mucosal barrier damage in a rat model of intestinal ischemia/reperfusion injury. J Surg Res 213: 75-83, 2017.

30. Ikeda H, Suzuki Y, Suzuki M, Koike M, Tamura J, Tong J, Nomura M and fItoh G: Apoptosis is a major mode of cell death caused by ischaemia and ischaemia/reperfusion injury to the rat intestinal epithelium. Gut 42: 530-537, 1998.

31. Shen JT, Li YS, Xia ZQ, Wen SH, Yao X, Yang WJ, Li C and Liu KX: Remifentanil preconditioning protects the small intestine against ischemia/reperfusion injury via intestinal $\delta$ - and $\mu$-opioid receptors. Surgery 159: 548-559, 2016.

32. Eltzschig HK and Eckle T: Ischemia and reperfusion-from mechanism to translation. Nat Med 17: 1391-1401, 2011.
33. Li LX, Yin LH, Gao M, Xu LN, Qi Y and Peng JY: MiR-23a-5p exacerbates intestinal ischemia-reperfusion injury by promoting oxidative stress via targeting PPAR alpha. Biochem Pharmacol 180: 114194, 2020.

34. Wu MB, Ma B,Zhang TX, Zhao K, Cui SM and He SC: Propofol improves intestinal ischemia-reperfusion injury in rats through NF-кB pathway. Eur Rev Med Pharmacol Sci 24: 6463-6469, 2020.

35. Yang X, Wan M, Cheng Z, Wang Z and Wu Q: Tofacitinib inhibits ox-LDL-induced adhesion of THP-1 monocytes to endothelial cells. Artif Cell Nanomed Biotechnol 47: 2775-2782, 2019.

36. Wang AL, Niu Q, Shi N, Wang J, Jia XF, Lian HF, Liu Z and Liu CX: Glutamine ameliorates intestinal ischemia-reperfusion injury in rats by activating the Nrf2/Are signaling pathway. Int J Clin Exp Pathol 8: 7896-7904, 2015.

37. Wang H, Cai D, Chen Z and Wang Y: GTS-21 promotes $\alpha 7$ $\mathrm{nAChR}$ to alleviate intestinal ischemia-reperfusion-induced apoptosis and inflammation of enterocytes. Med Sci Monit 26: e921618, 2020

38. Meng QT, Chen R, Chen C, Su K, Li W, Tang LH, Liu HM, Xue R, Sun Q, Leng Y, et al: Transcription factors Nrf2 and NF- $\kappa \mathrm{B}$ contribute to inflammation and apoptosis induced by intestinal ischemia-reperfusion in mice. Int J Mol Med 40: 1731-1740, 2017.

39. Lee EB, Fleischmann R, Hall S, Wilkinson B, Bradley JD, Gruben D, Koncz T, Krishnaswami S, Wallenstein GV, Zang C, et al: Tofacitinib versus methotrexate in rheumatoid arthritis. N Engl J Med 370: 2377-2386, 2014.

40. Palasik BN and Wang HM: Tofacitinib, the first oral janus kinase inhibitor approved for adult ulcerative colitis. J Pharm Pract: Sep 2, 2020 (Online ahead of print).

41. Liu W, Singh SR and Hou SX: JAK-STAT is restrained by notch to control cell proliferation of the drosophila intestinal stem cells. J Cell Biochem 109: 992-999, 2010.

42. Lu KH, Wu HH, Lin RC, Lin YC, Lu PW, Yang SF and Yang JS: Curcumin analogue L48H37 suppresses human osteosarcoma U2OS and MG-63 Cells' migration and invasion in culture by inhibition of UPA via the JAK/STAT signaling pathway. Molecules 26: 30, 2020.

43. Yue Y, Zhang Q, Wu S, Wang S, Cui C, Yu M and Sun Z: Identification of key genes involved in JAK/STAT pathway in colorectal cancer. Mol Immunol 128: 287-297, 2020.

44. Feng P, Zhang A, Su M, Cai H, Wang X and Zhang Y: Dexmedetomidine inhibits apoptosis of astrocytes induced by oxygen-glucose deprivation via targeting JAK/STAT3 signal pathway. Brain Res 1750: 147141, 2021.

45. Lin Z, Rn J and Shan X: Fish oils protects against cecal ligation and puncture-induced septic acute kidney injury via the regulation of inflammation, oxidative stress and apoptosis. Int J Mol Med 44: 1771-1780, 2019.

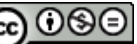

This work is licensed under a Creative Commons Attribution-NonCommercial-NoDerivatives 4.0 International (CC BY-NC-ND 4.0) License. 